\title{
Bosonization analysis for artificial "atomic collapse" in graphene
}

\author{
Aya Kagimura* \\ Particle Physics Theory Group, Department of Physics, Osaka university \\ E-mail: kagimuradhet.phys.sci.osaka-u.ac.jp

\section{Tetsuya Onog} \\ Particle Physics Theory Group, Department of Physics, Osaka university \\ E-mail: onogidhet.phys.sci.osaka-u.ac.jp
}

We report our non-perturbative study of the vacuum strucuture of the quasiparticles in graphene with a charge impurity. Using the bosonization method, which takes the multi body effect into account, we compute the induced charge screening. Our study will be useful to understand the electric property of the graphene and also give some insight to the "atomic collapse".

The 33rd International Symposium on Lattice Field Theory

14 -18 July 2015

Kobe International Conference Center, Kobe, Japan

${ }^{*}$ Speaker. 


\section{Problem and Formalism}

Graphene is the 2 dimensional crystal, discovered in 2004 [四]. It has honeycomb lattice structure. The electric structure of the graphene at low energy is known to be the same as that of the massless Dirac fermion. In addition, because the fermi velocity $v_{F}$ which plays the role of speed of light $c$ is $v_{F} \sim c / 300$, the effective fine structure constant $\alpha$ is about 300 times as large as that in the Quantum Electro Dynamics (QED). Due to this property, the essential point of the physics in the strongly coupled QED can be tested in the experiment using the graphene. Especially, we focus on the "atomic collapse" problem which is vacuum collapse due to strong Coulomb force. Putting charged impurities on the graphene, a system similar to the large $Z$ atom system can be realized, which enable us to observe the "atomic collapse".

The knowledge how the electron respond to the charged impurity in graphene is essential for utilization as a device. Therefore this system is well studied in one-particle theory as the system of the two dimensional massless electron in Coulomb potential [ [2, 目, 田]. It is predicted that when the charge of the impurity exceeds a critical value $Z_{c r}$, the wave function drastically changes. The massless fermion forms infinite number of quasibound states with negative energy, and the characteristic resonances appear in the local density of states (LDOS) of the electron[罒]. Inspired by these theoretical studies, the scanning tunneling microscope (STM) experiment was carried out and a characteristic peak in LDOS was measured[6].

In order to take the electron-electron interaction into the study of this problem, we start from the 3+1 QED with 2+1 dimensional Dirac massless fermion around an external charge:

$$
S=\int d^{4} x\left[-\frac{1}{4} F_{\mu v} F^{\mu v}+\delta(z) \bar{\psi}(i \partial+e A) \psi-Z e \rho(x) A_{0}\right]
$$

In order to analyze the system nonperturbatively, we employ the method proposed in ref.[W] for the atomic collapse QED with 3+1 dimensional massive fermion. See ref.[ए2] for details of our calculation for $2+1$ dimensional massless fermion.

We keep only spherically symmetric s-wave electromagnettic fields. Expanding the fermion field as angular momentum expansion, we can integrate the theory with the angle. Moreover, we keep only the lowest angular momentum $m= \pm 1 / 2$ mode of the fermion field, giving up higher angular momentum mode. Then the action becomes

$$
\begin{aligned}
S=\int d r d t & {\left[2 \pi r^{2}\left(\partial_{0} a_{1}-\partial_{r} a_{0}\right)^{2}-4 \pi Z \operatorname{Zer}^{2} \rho(r, t) a_{0}\right.} \\
& \left.+\sum_{m= \pm 1 / 2}\left[\bar{u}_{m}\left\{\gamma^{0}\left(i \partial_{0}+e a_{0}\right)+\gamma^{1}\left(i \partial_{r}+e a_{1}\right)\right\} u_{m}+i \frac{1}{2 r} \bar{u}_{m} \gamma^{5} u_{m}\right]\right] .
\end{aligned}
$$

$\rho(x)=\rho(r, t)$ is the charge density of spherical symmetric impurity, $a_{i}$ correspond the s-wave gauge field, and $u_{m}$ is the 2-component fermion field. The last term represents centrifugal force. We have to set the boundary condition for fermion field $u_{m}$ at $r=0$ by requiring single-valuedness at $r=0$. In terms of $2 \mathrm{D}$ fermion $u_{m}$, the boundary condition is

$$
\left(1-\gamma^{0}\right) u_{m}(0, t)=0 .
$$


We apply bosonization to this theory. Regarding interaction term as perturbation, we bosonize free fermion field to free boson field,

$$
u_{m}(r, t)=\left(\frac{\mu}{2 \pi}\right)^{1 / 2}\left(\begin{array}{c}
-i N_{\mu} \exp \left[i \sqrt{\pi}\left(\phi_{m}(r, t)+\tilde{\phi}_{m}(r, t)\right)\right] \\
N_{\mu} \exp \left[i \sqrt{\pi}\left(-\phi_{m}(r, t)+\tilde{\phi}_{m}(r, t)\right)\right]
\end{array}\right),
$$

where

$$
\tilde{\phi}(x)=\lim _{\varepsilon \rightarrow 0} \int_{r}^{\infty} d s e^{-\varepsilon s} \dot{\phi}(s, t),
$$

and $N_{\mu}$ represents normal ordering at IR mass scale $\mu$. From now on, we use the overdot and prime for time and spatial derivative, respectively. In this case, we should impose the boundary condition on the boson field. The boundary condition $(\mathbb{L} .3)$ is rewritten in boson field as

$$
\phi_{m}(0, t)=0 .
$$

By careful treatment of the unusual commutation relation due to this boundary condition, the interaction terms in fermion theory is mapped into boson field interaction terms. After some point splitting procedure, we can write the action (ㅍ.2) in terms of boson operators. We take $a_{1}=0$ gauge, and $a_{0}$ can be integrated out. Then the Hamiltonian becomes

$$
\begin{gathered}
H=\int d r\left[\sum_{m= \pm 1 / 2}\left\{\frac{1}{2}\left(\pi_{m}^{2}+\phi_{m}^{\prime 2}\right)+\frac{1}{4 \pi r^{2}}\left(1-\cos \left(2 \sqrt{\pi} \phi_{m}\right)\right)\right\}\right. \\
\left.+\frac{e^{2}}{8 \pi r^{2}}\left\{\left(\Phi(r, t)-\frac{1}{\sqrt{\pi}} \sum_{m} \phi_{m}\right)^{2}-\Phi(r, t)^{2}\right\}\right],
\end{gathered}
$$

where the $\Phi(r, t)$ is defined by

$$
\Phi^{\prime}(r, t)=4 \pi Z r^{2} \rho(r, t) .
$$

We add the c-number so that the energy becomes zero when $\phi_{m}=0$ which is vacuum configuration with $Z=0$.

\section{Study of vacuum solution}

In this section we find the classical solution which minimizes bosonized Hamiltonian (ㅍ.]). In order to study the vacuum structure, we consider only static solution $\pi_{m}=0$. We assume that the impurity charge is spherically spread over radius $R$. Then the corresponding $\Phi(r)$ becomes

$$
\Phi(r)=\left\{\begin{array}{cc}
Z\left(\frac{r}{R}\right)^{3} & (r<R) \\
Z & (r>R) .
\end{array}\right.
$$

Rewriting the Hamiltonian in terms of the new variable $\phi_{ \pm}=\frac{1}{\sqrt{2}}\left(\phi_{+1 / 2} \pm \phi_{-1 / 2}\right)$, we notice that $\phi_{-}=0$ satisfies the Euler-Lagrange equation. So we take the symmetric ansatz $\phi_{-}=0$. Then the Euler-Lagrange equation for $\phi_{+}$is

$$
\phi_{+}^{\prime \prime}-\frac{1}{\sqrt{2 \pi} r^{2}} \sin \left(\sqrt{2 \pi} \phi_{+}\right)-\frac{2 \alpha}{\pi r^{2}}\left(\phi_{+}-\sqrt{\frac{\pi}{2}} \Phi(r)\right)=0 .
$$




\subsection{Asymptotic behavior}

Since Eq.(2.2) is a second order differential equation, in addition to the boundary condition at the origin we need to impose another boundary condition at $r=\infty$. For finiteness of total energy, the boson field should asymptotically be constant value $\phi_{*}$ at large $r$. Substituting $\phi_{+}=\phi_{*}$ into the Euler-Lagrange equation at large $r$, we find that $\phi_{*}$ should be the solution of following equation:

$$
\sin \left(\sqrt{2 \pi} \phi_{*}\right)=-2 \alpha\left(\sqrt{\frac{2}{\pi}} \phi_{*}-Z\right) .
$$

Total induced charge which screens the impurity charge is given by the asymptotic value of the boson field:

$$
Q_{\mathrm{EM}}=-e \sqrt{\frac{2}{\pi}} \phi_{*}
$$

Notice that the asymptotic value $\sqrt{\frac{2}{\pi}} \phi_{*}$ can take non-integer value. Charge screening with noninteger charge may seem counter intuitive if one tries to interpret the phenomena as particle hole pair creation. One should interpret such screening as the polarization effect.

Starting from the asymptotic solutions and solving the differential equation numerically, we can obtain the full solution. Taking the following asymptotic form $\phi_{+}(r) \approx \phi_{*}-\frac{A}{r^{\lambda}}$ at large $r$ and varying $A$, we can search for the physical solution which satisfies the boundary condition at $r=0$. For illustration, we show the example of $Z=4$ and $\alpha=0.2$; Fig.W. In this case, there are three asymptotic solutions, but only the solution which realizes the smallest value of $\phi_{*}$ can satisfy the appropriate boundary condition. The full solutions from the other asymptotic form does not satisfy the boundary condition at $r=0$ but end up have positive values no matter how we choose the value of $A$.

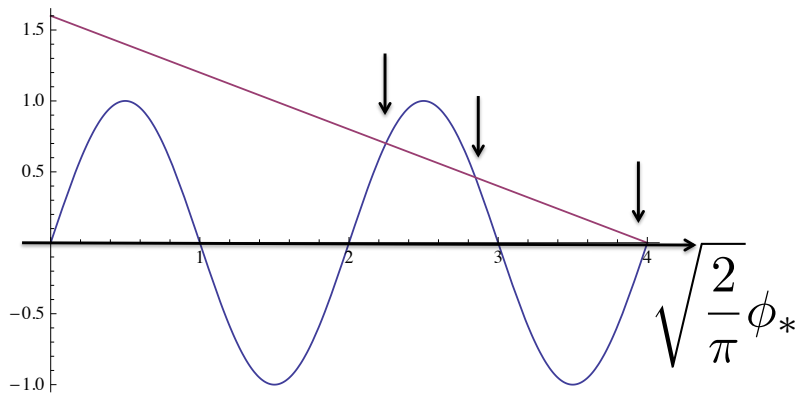

Figure 1: The blue line is 1.h.s. of eq.([2.3]), and red line is r.h.s. of eq.([2.3]) with $Z=4, \alpha=0.2$.

\subsection{Result}

We looked for the solutions for various set of parameters of $(\alpha, Z)$, where the parameter set is given in [Fig. [B]. We found that only the solution with the smallest value of $\phi_{*}$ can satisfy correct boundary condition at $r=0$ in all cases. From this fact, we reach the conjecture that the magnitude of screening can be determined by the smallest intersection of $\sin \left(\sqrt{2 \pi} \phi_{*}\right)$ and $-2 \alpha\left(\sqrt{2 / \pi} \phi_{*}-Z\right)$. 
According to this conjecture, we get effective impurity charge seen from infinitely separated point,

$$
Z_{\mathrm{eff}}=Z-\sqrt{\frac{2}{\pi}} \phi_{*}
$$

which is screened by induced charge [Fig.Z]. Notice that when $\alpha \gtrsim 0.2$, the effective impurity charge $Z_{\text {eff }}$ in any odd $Z$ case is the same one as in $Z=1$ case. Also when $\alpha \gtrsim 0.14, Z_{\text {eff }}$ in any even $Z$ case is the same one as in $Z=2$ case. From this result, a phase diagram of screening is described as [Fig.B]]. In larger $Z$ case, more branches appear in small $\alpha$ regime.
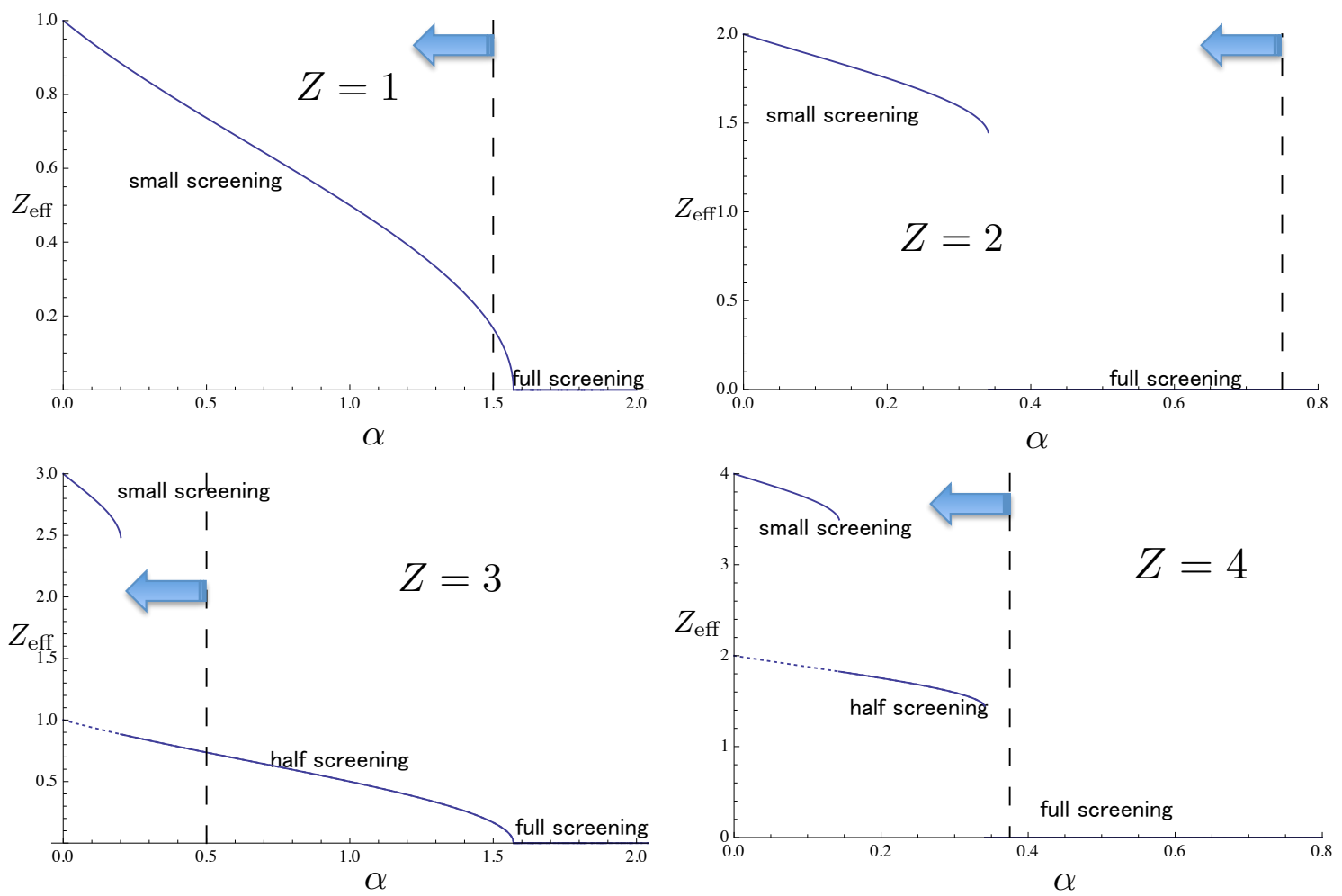

Figure 2: $Z_{\text {eff }}$ for each $Z$. Dotted lines in $Z=3,4$ cases describe $Z_{\text {eff }}$ in $Z=1,2$ cases, respectively. Dashed vertical lines describe the point $Z \alpha=3 / 2$ which may spoil the lowest partial wave approximation.

We can calculate the induced 2D electron density from full solution of the boson field. We fit the scaling law

$$
n(r) \propto r^{-\gamma}
$$

in the range of distances $1 \ll r / R \ll 10$. If graphene sheet can be treated as perfect metal, the scaling law is calculated in ref.[[3]] : $n(r) \propto r^{-3}$ in the range of distances $1 \ll r / R \ll 2 \alpha^{2} Z$. In our calculation, The scaling exponent $\gamma$ depends on parameters $\alpha, Z$ as shown in [Fig.团]. In small screening regime, we get $\gamma \sim 2.7$, independently of $\alpha$. Near the value of $\alpha$ where magnitude of screening jumps, $\gamma$ drastically decreases. In larger $\alpha$ regime, $\gamma$ increases and becomes close to $\gamma=3$ which is the value calculated in perfect metal approximation. 


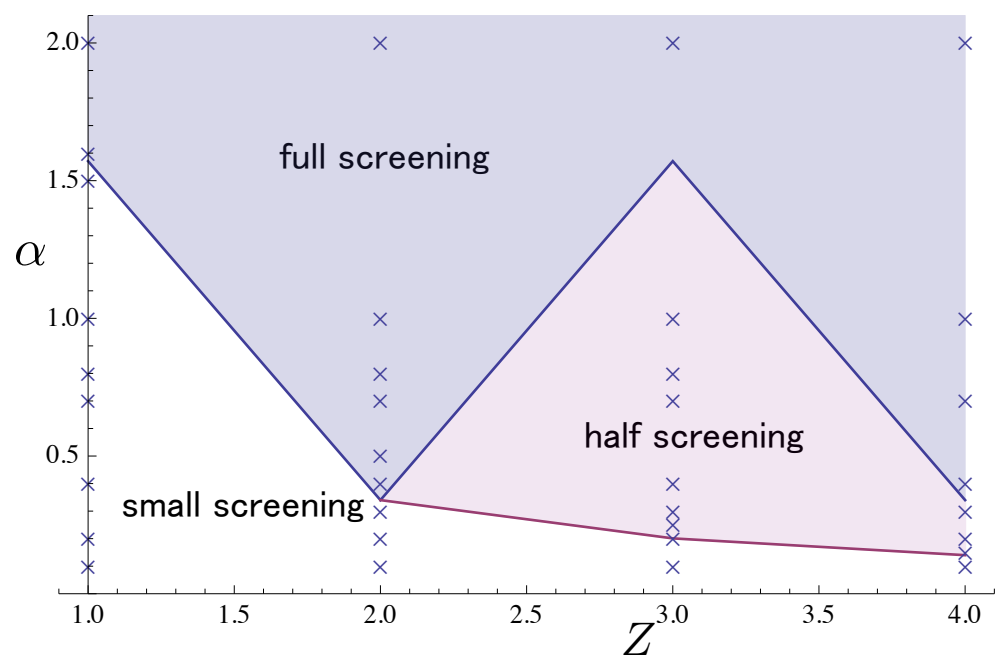

Figure 3: Phase diagram of screening. Crosses are the parameter point where actually we solve the equation (2.2) in whole $r$.
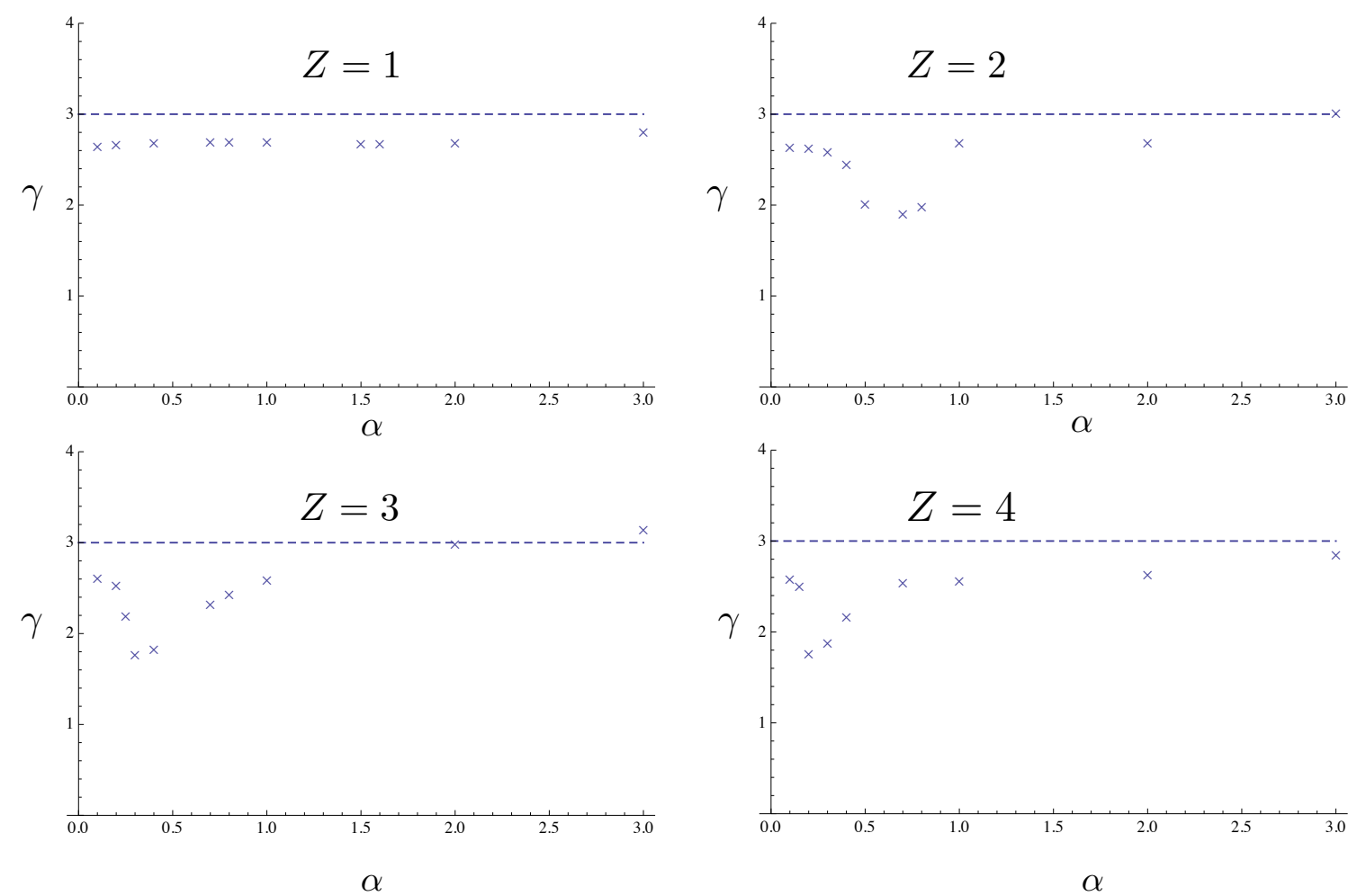

Figure 4: Crosses describe the scaling exponent in each $Z$ case. Dashed line describe one in perfect metal approximation. 


\section{Summary}

In this proceeding, we presented our study on quantum field theory with the $2+1$ dimensional massless fermion around an external Coulomb field. We reduced the theory to a two dimensional fermion theory, where the higher partial wave are neglected. Bosonizing the theory, we have found the static solution of classical equation of motion for the boson field. The magnitude of screening is determined only by the asymptotic equation of motion. Which of these asymptotic solution satisfies the boundary condition at $r=0$ is determined by the dynamics.

Through the study of several examples, we have concluded that the realized solution is always the smallest screening one. As a result, we have found patterns of screening depending on the coupling $\alpha$ and the impurity charge $Z$. The screening charge undergoes a drastic change as we change the value of $\alpha$ at some critical values. By solving the equation of motion in full spatial range, we have obtained the spatial distribution of density of the induced electron. The radial profile of the two dimensional induced charge density can be fitted by negative power in $r$ which is the distance from the impurity.

The validity of the approximation to neglect higher partial wave can be discussed somewhat in semi classical theory mentioned in section 2 . According to the semi classical theory, only $Z \alpha>j$ wave can form quasi-bound states. So, the fermion mode whose angular momentum $j$ is higher than $Z \alpha$ is irrelevant to anomalous behavior of the electron in strong Coulomb potential. When $Z \alpha>3 / 2$, the next to lowest partial wave $j=3 / 2$ should be relevant to this problem. Therefore our approximation should be valid only when $Z \alpha<3 / 2$.

\section{References}

[1] K.S. Novoselov, A.K. Geim, S.V. Morozov, D. Jiang, Y. Zhang, S.V. Dubonos, I.V.Grigorieva, A.A. Firsov, Science 306(2004) 666.

[2] Vitor M. Pereira, Johan Nilsson, and A. H. Castro Neto, Phys. Rev. Lett. 99 (2007) 166802.

[3] A. V. Shytov, M. I.Katsnelson, L. S. Levitov, Phys. Rev. Lett. 99 (2007) 236801.

[4] Y. Nishida, Phys. Rev. B 90 (2014) 165414.

[5] A. V. Shytov, M. I.Katsnelson, L. S. Levitov, Phys. Rev. Lett. 99 (2007) 246802.

[6] Wang, Yang, et al. Science 340 (2013) 734.

[7] S. R. Coleman, Phys. Rev. D 11 (1975) 2088.

[8] S. Mandelstam, Phys. Rev. D 11 (1975) 3026.

[9] C. G. Callan, Jr., Phys. Rev. D 26 (1982) 2058.

[10] J. A. Harvey, Phys. Lett. B 131 (1983) 104.

[11] Y. Hirata and H. Minakata, Phys. Rev. D 34 (1986) 2493.

[12] A. Kagimura and T. Onogi, arXiv:1508.00180 [hep-th].

[13] M. M. Fogler, D. S. Novikov and B. I. Shklovskii Phys. Rev. B 76 (2007) 233402. 